\title{
Avidity of anti-phospholipid antibodies in relation to their levels
}

\author{
LENKA FIALOVÁ, OLIVER KUCHAŘ, MILADA PETRÁČKOVÁ, IVAN MALBOHAN, TOMÁŠ ZIMA
}

Institute of Medical Biochemistry and Laboratory Diagnostics, First Faculty of Medicine, Charles University and General University Hospital in Prague, Czech Republic

\begin{abstract}
Introduction: The heterogeneity of anti-phospholipid antibodies can be manifested not only in different antigenic specificities, but also in their avidities. The aim of the study was to investigate the relationship between anti-cardiolipin antibody $(a C L) \operatorname{Ig} G$ avidities and levels within the range of their titres, from very low to high ones.

Material and methods: We analyzed 78 serum samples from 60 patients by ELISA with chaotropic agents, using urea concentration of 6 and $8 \mathrm{~mol} / \mathrm{l}$ and single diluted serum samples. The changes of aCL levels and avidities were explored during a long-term follow-up in 14 patients.

Results: The avidities of aCLs did not differ in the groups of patients classified according to aCL levels. The higher avidity antibodies predominated in our patients and the fluctuation of avidities in the longitudinal follow-up did not show significant differences. No relationship between aCL levels and their avidities was found.

Conclusions: aCL avidities seem to have no relationship with aCL levels and high-avidity aCLs; the potentially deleterious effects might be present also in patients with low and extremely low aCL levels. Avidity of aCLs belongs to stable characteristics with insignificant changes in time.
\end{abstract}

Key words: anti-cardiolipin antibodies, anti-phospholipid antibodies, avidity, ELISA, chaotropic agents, urea, thrombosis, systemic lupus erythematosus.

(Cent Eur J Immunol 2020; 45 (2): 136-143)

\section{Introduction}

Anti-phospholipid antibodies (aPLs) include a very heterogeneous group of autoantibodies, directed against a variety of antigenic targets $[1,2]$. Persistently elevated levels of aPLs represent important laboratory findings in the anti-phospholipid syndrome (APS), an autoimmune disease clinically manifested by vascular thrombosis and/ or pregnancy complications [3-5]. According to the international classification criteria for APS anti-cardiolipin antibodies (aCLs) IgG/IgM, anti- $\beta 2$-glycoprotein I antibodies (aß2GPI) IgG/IgM or lupus anti-coagulant are involved as laboratory criteria for APS [6].

Because not all of the patients who display clinical symptoms of APS produce anti-phospholipid antibodies as listed in current APS criteria, new approaches in aPL testing have been intensively studied [7-9]. The inclusion of other aPLs into the APS criteria is considered. Anti-prothrombin, anti-phosphatidylserine/prothrombin, or aß2GPI domain 1 antibodies are examples of antibodies, so-called non-criteria aPLs $[9,10]$. However, the heterogeneity of antibodies can be expressed not only in different antigenic specificity, but also in their avidity. It is an important characteristic feature of antibodies, which describe the total binding strength between an antibody and an antigen [11]. Antibody avidities used to be examined in the antibodies directed against infectious agents, with the aim to evaluate their avidity maturation associated with the increase of specific humoral immunity [12,13]. Avidities of autoantibodies including aPLs have already been investigated [14-19]. In contrast to anti-infectious antibodies, the autoantibody avidities can reach different values. High, low, or moderate avidity of autoantibodies occurs in autoimmune diseases. Nevertheless, it is supposed that irrespective of their avidities, autoantibodies can participate in the pathogenesis of some autoimmune diseases [20, 21].

In this pilot study, we focused on obtaining more detailed information about aCL IgG avidity. According to our knowledge, the avidity of aCLs had been explored in patients with positive aCLs, mostly in those with APS, systemic lupus erythematosus (SLE), autoimmune liver diseases, and HIV-1-infected patients [22-25]. We were interested not only in the avidity of positive aCLs, but we also analyzed if aCL avidities were connected to aCL with 
low levels. We paid attention to patients with low and very low levels of aCLs, considering the subjects of the $14^{\text {th }}$ International Congress on Antiphospholipid Antibodies Task Force [26]. Authors of a recent study suggested that a decrease of the aPL level threshold in APS criteria could be more suitable for risk stratification of cardiovascular accident among healthy persons [27].

In the present study, we investigated the relationship between aCL IgG avidities and levels within the range of their levels, from very low to high ones, and hypothesized that very low levels of aCL might be characterized by higher avidity and that these high-avidity aCLs may be present in symptomatic patients. Moreover, we evaluated the changes of aCL levels and avidities in the group of patients during a long-term follow-up. We also examined whether the avidity of aCLs was related to levels of non-criteria types of aPLs.

\section{Material and methods}

\section{Patients}

We retrospectively analyzed 78 serum samples from 60 patients (age, $48 \pm 19$ years; mean $\pm S D$; sex, 36 females, 24 males). Collection of samples was conducted at the Immunological Department of the Institute of Medical Biochemistry and Laboratory Diagnostics in Prague (Czech Republic) in the period from 2011 to 2015 and in the Thomayer Hospital (Prague, Czech Republic) between 2015 and 2016. The patients were examined or hospitalized for systemic immunological diseases $(n=14$, predominantly with systemic lupus erythematosus) and venous thrombosis $(n=18)$. The diagnosis of deep venous thrombosis was made using patients' history, physical evaluation, and Doppler-sonography in particular. None of the studied patients had a malignant disease. The rest of the patients $(n=28)$ included mainly those with miscellaneous diseases, such as renal (glomerulonephritis or tubulointerstitial nephritis), gastrointestinal disorders (predominantly pancreatitis and biliary disease, such as cholelithiasis), or immunodeficiency, female infertility, and diabetes mellitus. The diagnoses were determined by experienced internists based on valid diagnostic criteria.

All subjects provided written informed consent regarding participation in the study. The ethics committees of the General University Hospital, Prague and Thomayer Hospital, Prague, have approved the study.

\section{Methods}

\section{Levels of various types of anti-phospholipid antibodies}

aCL IgG levels were determined in the sera by ELISA kits (Orgentec, Mainz, Germany). Similarly, other types of anti-phospholipid antibodies directed against other structures (anti- $\beta 2$-glycoprotein I, anti-phosphatidylserine, an- ti-phosphatidylinositol, anti-phosphatidic acid) were also analyzed by ELISA kits (Orgentec, Mainz, Germany) in patients from the Immunological Department. According to the manufacturer recommendation, the cut-off values for aPLs were 10 GPL. Coefficients of variations for intra- and inter-assay precision of ELISAs declared by the manufacturer were mostly less than $5 \%$ and never exceeded $10 \%$. In general, the measuring range for the aCL ELISA kit is from 0 to $120 \mathrm{GPL}$, with a functional sensitivity of $1 \mathrm{GPL}$.

\section{Avidity of aCL IgG}

The aCL IgG avidity was determined by the modified ELISA method presented by Vlachoyiannopoulos et al. [23]. In our previous study, we investigated the possibilities of aCL avidity determination using chaotropic agents in detail. We studied the most appropriate dilution of sera and concentration of urea in our modification of ELISA method for aCL avidity determination. The urea concentration of $2 \mathrm{~mol} / \mathrm{l}$ and $4 \mathrm{~mol} / \mathrm{l}$ did not disassociate the immune complexes sufficiently, especially if the aCL levels were higher. We found that both urea in concentrations of $6 \mathrm{~mol} / \mathrm{l}$ or $8 \mathrm{~mol} / \mathrm{l}$ and sodium chloride in concentrations of $1 \mathrm{~mol} / \mathrm{l}$ or $2 \mathrm{~mol} / \mathrm{l}$ were suitable for the sufficient dissociation of immune complexes during an extra step in the ELISA procedure. The extended description of the ELISA for avidity examination using chaotropic agents is shown in Fialová et al. [17, 18]. For the purpose of this study, we used sera diluted 1:50 and urea in concentrations of $6 \mathrm{~mol} / \mathrm{l}$ and $8 \mathrm{~mol} / \mathrm{l}$ as a chaotrope. The results of aCL IgG avidity were expressed as the avidity index (AI). It was calculated as a ratio (or percentage) of the residual antibodies bound in the wells in the presence of chaotropic solution $(6 \mathrm{~mol} / \mathrm{l}$ urea or $8 \mathrm{~mol} / \mathrm{l}$ urea) to the total antibodies bound in the absence of a chaotrope.

$$
\mathrm{AI}=\frac{\text { absorbance in the wells with chaotrope }}{\text { absorbance in the wells without chaotrope }}
$$

The antibodies were classified as high-avidity ones, when the avidity index was higher than 0.6 and low-avidity ones, when the avidity index was lower than 0.4 . The values of the avidity index of the antibodies from 0.4 to 0.6 were usually marked as a "grey zone" [28].

\section{Statistics}

Since the data did not express normal distribution, non-parametric statistical tests were used. The comparison of groups was performed by the Kruskal-Wallis test. The Spearman correlation coefficient was applied for correlation analyses. The paired measurements were evaluated by the Wilcoxon matched pairs test. The level of statistical significance was set as $p=0.05$. Statistical analyses were performed using MedCalc (Ostend, Belgium) and Statistica programs (TIBCO Software Inc., ArcIT Consulting s.r.o., Czech Republic). 


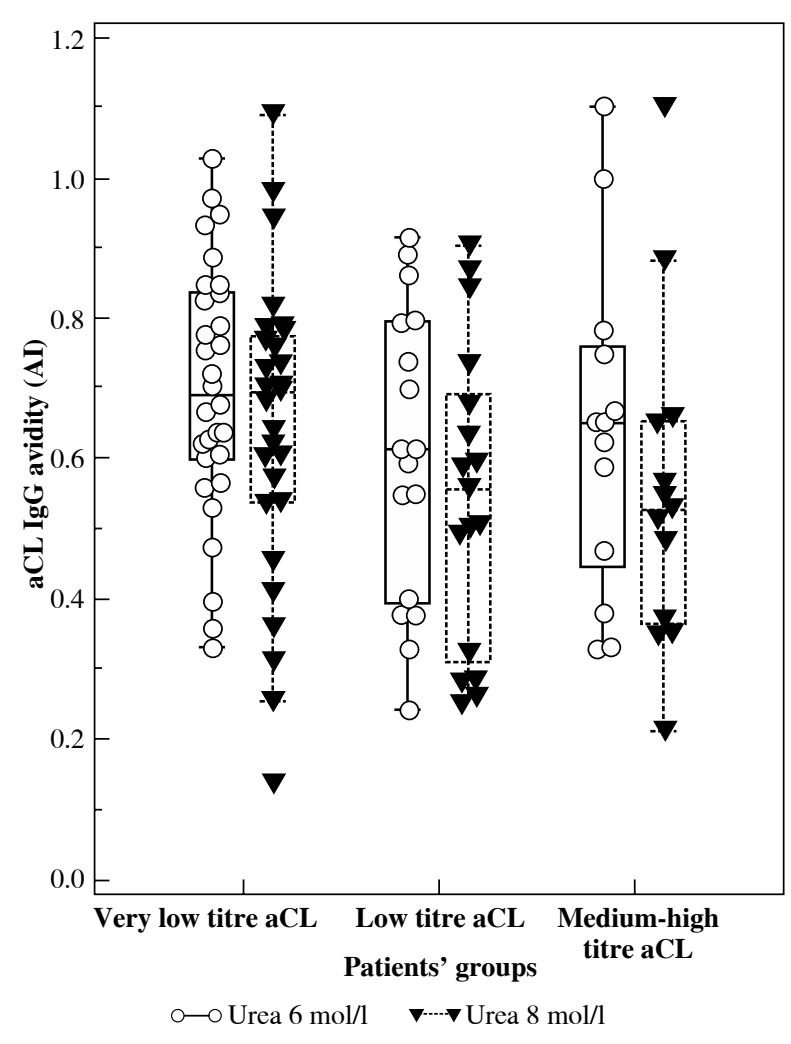

Fig. 1. aCL IgG avidities in the groups of patients classified according to aCL titre (very low titre $\mathrm{aCL}<10 \mathrm{GPL}$, $n=30$; low titre aCL 10-40 GPL, $n=17$; medium-high titre aCL > 40 GPL, $n=13$ ). No significant difference was found among groups regardless of urea concentration used for avidity determination. The central box represents the values from the lower to upper quartile $\left(25^{\text {th }}\right.$ to $75^{\text {th }}$ percentile). The median is shown as the middle line. Outside and far out values are displayed as separate points.

\section{Results}

\section{Comparison of aCL IgG avidities according to aCL IgG levels}

Patients were divided according to aCL IgG levels into three groups: very low level ( $<10$ GPL; GPL - standardized international units; $\mathrm{n}=30$ ), low level (10-40 GPL; $\mathrm{n}=17$ ), and medium-high level (> $40 \mathrm{GPL} ; \mathrm{n}=13)$. The value of aCL IgG higher than $40 \mathrm{GPL}$ are designated in laboratory criteria for APS as a cut-off for medium to high levels (titers) [6, 29], and value of 10 GPL is a threshold for positive and negative outcomes declared by manufacturers for positive and negative results. If more than one sample was obtained from a patient, the serum values of the first blood sampling were evaluated. Except for one subject, no patient with thrombosis presented aCL level
$>10$ GPL. SLE or systemic connective tissue disease have been diagnosed in 11 patients, with aCL levels higher than 40 GPL. Figure 1 shows the values of avidity tested in the presence of urea with $6 \mathrm{~mol} / \mathrm{l}$ and $8 \mathrm{~mol} / \mathrm{l}$. The avidity of aCLs did not significantly differ among groups regardless of the urea concentration used for the determination of avidity. Medians of AI were above 0.6 for all groups using urea $6 \mathrm{~mol} / \mathrm{l}$ in the avidity analysis. An elevation of urea concentration for avidity determination resulted in a moderate decrease of AI medians, but no significant differences among patients' groups were found.

\section{Longitudinal evaluation of aCL IgG avidities}

A repeated blood sampling was conducted in 14 patients in the interval of 315 days (range, 98-392 days; median $25^{\text {th }}-75^{\text {th }}$ percentile). Characteristics of longitudinally evaluated patients (age, $43 \pm 15$ years; mean \pm SD) are presented in Table 1. Paired serum samples were obtained from most patients, except for three patients, who were sampled three or four times. Patients with immunological diseases prevail in this group. The proportion of patients with low or medium-high levels of aCLs was similar among patients. The AI value of aCLs exceeded 0.6 in most patients. The aCL levels as well as their avidities did not significantly change during follow-up (Fig. 2). The time of development of levels and avidities of aCLs in three patients, who were sampled more than twice, are demonstrated in Figure 3. It is obvious that the changes in aCL levels were not uniformly accompanied by similar changes in their avidities. However, the observed changes in aCL level did not exceed the range typical for the category of aCL level, such as low or medium-high aCL levels. Similarly, the variations in aCL avidity, except for patient A, were irrelevant.

\section{Correlation analyses}

The aCL avidities determined using urea $6 \mathrm{~mol} / \mathrm{l}$ or $8 \mathrm{~mol} / \mathrm{l}$ did not correlate with their levels in any of the groups of patients classified according to aCL levels (very low, low, and medium-high aCL level groups). On the contrary, the aCL levels significantly correlated with the levels of aPLs directed against $\beta 2$ GPI and other phospholipids, but not with the aCL avidities (Table 2).

\section{Discussion}

In our pilot study, we investigated the relationship between avidities and aCL IgG levels across the range of their levels from very low to high ones, using the ELISA method with chaotropic agents. The aCL avidities did not differ in the groups of patients classified according to aCL levels. Higher avidity antibodies predominated in our patients, and the fluctuation of avidities in the longitudinal 
Table 1. Clinical characteristics of repeatedly examined patients

\begin{tabular}{|c|c|c|c|c|c|}
\hline \multicolumn{4}{|c|}{ Basic characteristics of patients } & \multicolumn{2}{|c|}{ aCL IgG avidity follow-up changes } \\
\hline $\begin{array}{l}\text { Patient } \\
\text { no. }\end{array}$ & Diagnosis & $\begin{array}{l}\text { Age (years), } \\
\text { sex }\end{array}$ & $\begin{array}{l}\text { Number of } \\
\text { repeated aCL } \\
\text { examinations }\end{array}$ & $\begin{array}{l}\text { aCL IgG avidity } \\
\text { (6 mol/l urea) in AI } \\
\text { follow-up change }\end{array}$ & $\begin{array}{c}\text { aCL IgG avidity } \\
(8 \mathrm{~mol} / \mathrm{l} \text { urea) in AI follow- } \\
\text { up change }\end{array}$ \\
\hline 1 & Sjögren syndrome & 71, female & 2 & $0.74-0.70$ & $0.67-0.63$ \\
\hline 2 & SLE & 46 , male & 3 & $0.67-0.73-0.58$ & $0.53-0.64-0.3$ \\
\hline 3 & SLE & 41 , male & 3 & $0.61-0.66-0.63$ & $0.59-0.69-0.56$ \\
\hline 4 & SLE & 41 , female & 2 & $0.4-0.65$ & $0.32-0.56$ \\
\hline 5 & SLE & 38 , female & 2 & $0.65-0.34$ & $0.54-0.32$ \\
\hline 6 & Systemic connective tissue disease & 35 , female & 2 & $0.37-0.57$ & $0.21-0.481$ \\
\hline 7 & Systemic connective tissue disease & 33 , female & 2 & $0.33-0.26$ & $0.32-0.26$ \\
\hline 8 & Coagulation disorder & 64 , female & 2 & $0.79-0.82$ & $0.84-0.72$ \\
\hline 9 & Coagulation disorder & 15 , male & 2 & $0.64-0.65$ & $0.64-0.48$ \\
\hline 10 & Immunodeficiency & 30, female & 2 & $0.86-0.68$ & $0.58-0.38$ \\
\hline 11 & Immunodeficiency & 30 , female & 4 & $0.33-0,56-0.64-0.47$ & $0.37-0.48-0.44-0.36$ \\
\hline 12 & Female infertility & 33 , female & 2 & $0.89-0.84$ & $0.90-0.72$ \\
\hline 13 & Defect of LFA-1 & 62 , female & 2 & $0.79-0.57$ & $0.63-0.47$ \\
\hline 14 & Glomerular abnormality & 56 , female & 2 & $0.59-0.65$ & $0.56-0.49$ \\
\hline
\end{tabular}

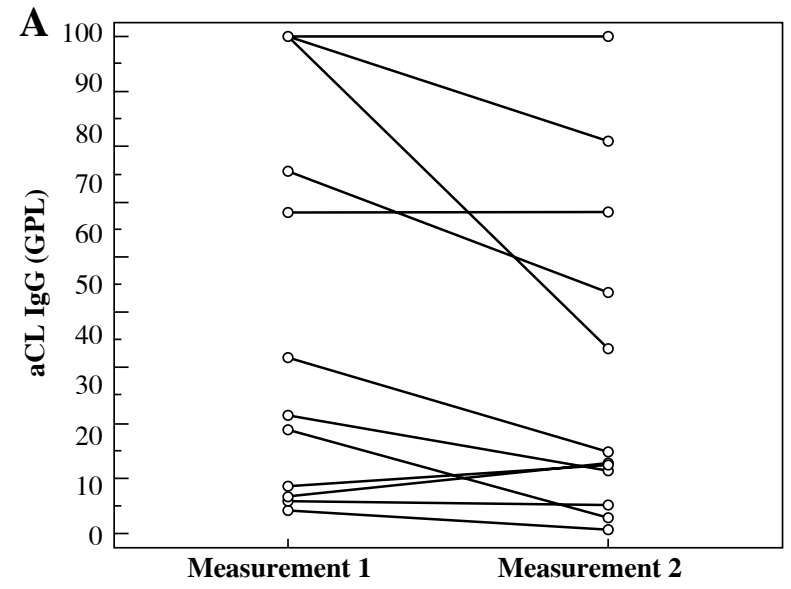

Fig. 2. Dynamics of aCL IgG titres and avidities in 14 patients during follow-up. A) Changes in aCL IgG levels; B) Changes of aCL IgG avidities using $6 \mathrm{~mol} / \mathrm{l}$ urea for avidity determination; C) Changes of aCL IgG avidities using $8 \mathrm{~mol} / \mathrm{l}$ urea for avidity determination. Neither aCL IgG levels, nor their avidities determined by $6 \mathrm{~mol} / \mathrm{l}$ urea as well as $8 \mathrm{~mol} / \mathrm{l}$ urea significantly differ during follow-up
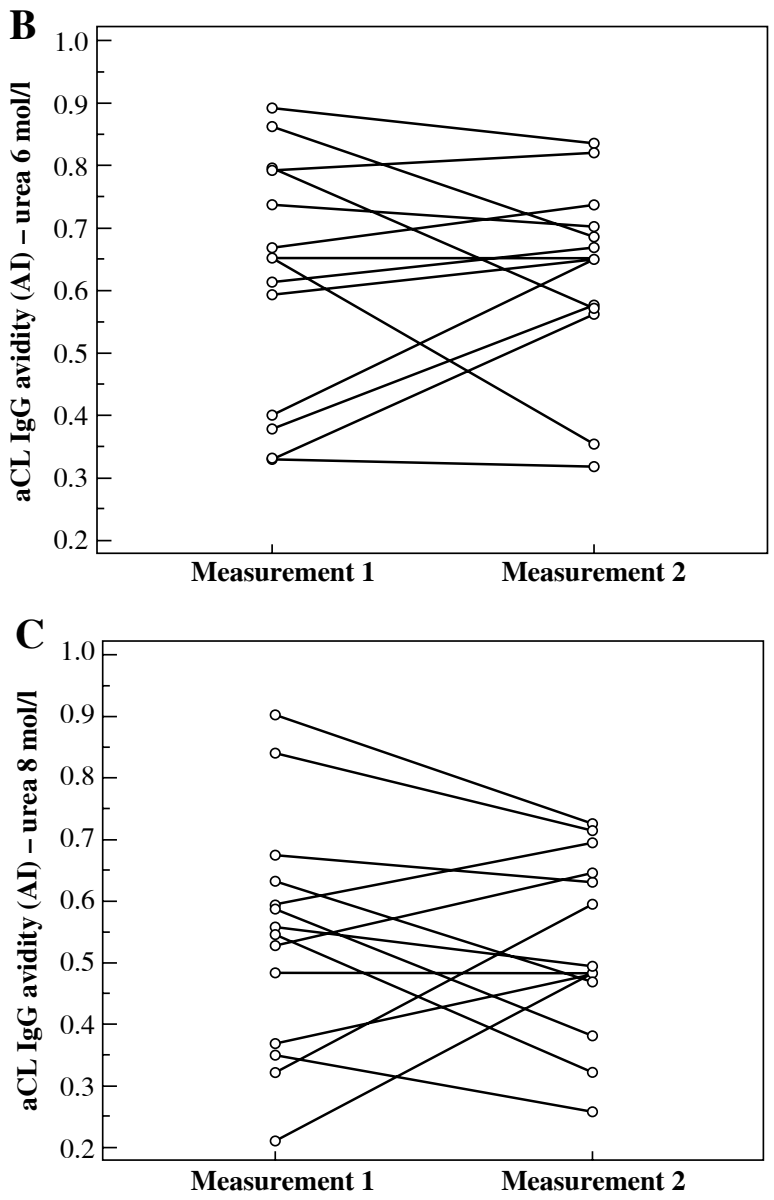

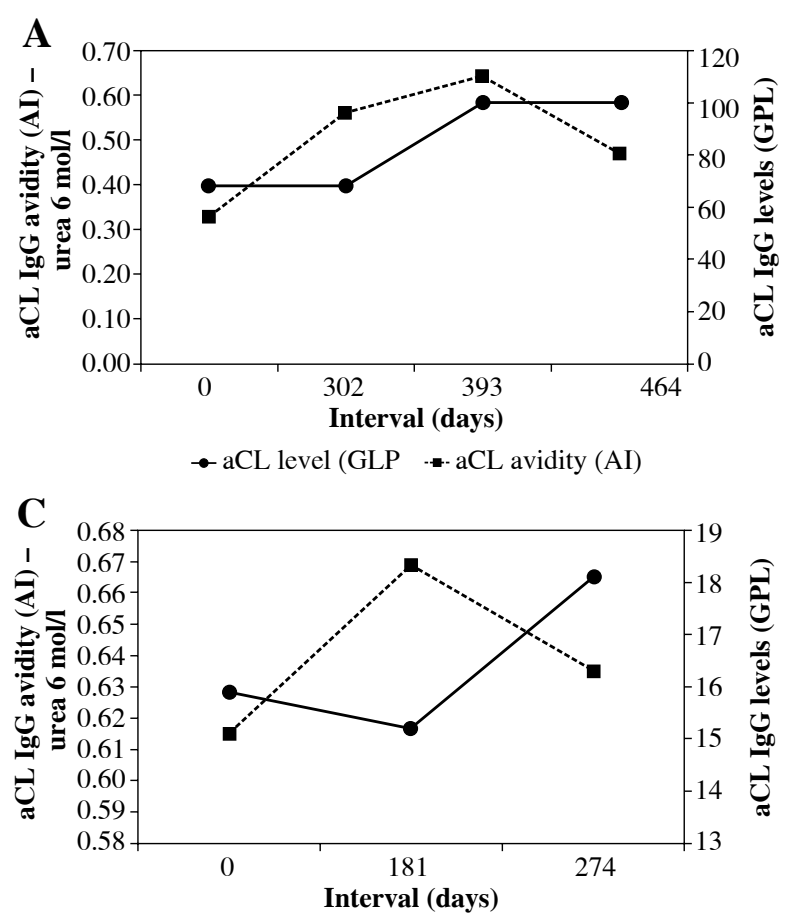

$\rightarrow$ aCL level (GLP - aCL avidity (AI)

follow-up did not show significant differences. In addition, no relationship was found between aCL levels and avidities in our patients.

A variety of methods and their modification for antibody avidity has already been described $[11,30]$. Recently, we explored in detail aCL IgG avidity methods using the principle of ELISA with chaotropes, which break the interactions of low-avidity antibodies with antigens [18]. A higher concentration of chaotropic agents caused more effective disruption of immune complexes. In this study, we applied a simple modification of the method used in the analysis of a single diluted serum exposed to a solution of urea. In our previous study [18], we found a good correlation between results obtained with a urea concentration of 6 or 8 $\mathrm{mol} / \mathrm{l}$. Nevertheless, with regards to an inclusion of serum samples with very high levels of aCLs, we performed the avidity analyses in the condition of both urea concentrations. However, independently of the used urea concentration in avidity analyses, significant differences in AI medians were not observed among tested patient groups.

Higher-avidity aCLs prevailed in our patients, since AI medians in all groups were above the value of 0.6 using urea $6 \mathrm{~mol} / \mathrm{l}$ as the chaotrope. It is not surprising, because healthy persons were not included for testing. It is wellknown that antibodies synthesized early in the immune response are characterized by low affinity, but the process of affinity maturation depends on many circumstances [31]. The initiation of autoimmune process is not precisely known, and many years without symptoms can precede clinical manifestation of autoimmune diseases. During

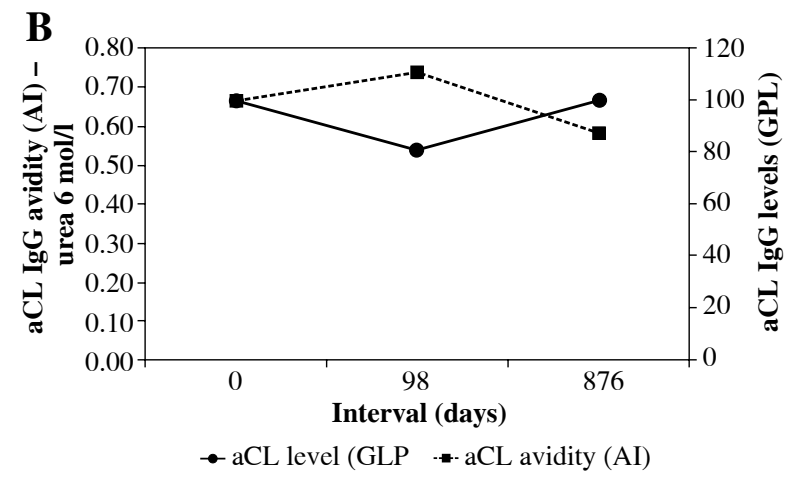

Fig. 3. Longitudinal follow-up of aCL IgG avidities and titres of in three patients. A) Patient A (female, 30 years old at the first blood sampling) was monitored for immunodeficiency; B) and C) two other patients were treated for systemic lupus erythematosus (B: male, 46 years old at the first blood sampling; $\mathrm{C}$ : male, 41 years old at the first blood sampling) (aCL - anti-cardiolipin antibody, AI - avidity index, GPL - standardized international units)

this period, when the autoimmune humoral response is enhanced, levels and avidities of autoantibodies can increase [32]. The avidities of autoantibodies in autoimmune diseases studied to date have mostly reached higher values, and can participate in the severity of disease $[15,16$, 33]. However, the nature of antigen seems to influence the maturation of antibody avidity. For example, avidities of anti-amyloid-b antibodies in Alzheimer's disease were characterized by lower avidities [34]. An interesting comparison between avidities of antibodies against recall antigens and autoantigens suggested that regulation of immune B cell response can differ [35-37]. While the avidities of autoantibodies against citrullinated and carbamylated proteins, or tranglutaminase- 2 tend to be low, avidities against recall antigens, such as tetanus toxoid, diphtheria toxoid, or gliadin, can be high despite their low titre. Van Delft et al. [37] explained low avidity of autoantibodies as a result of chronic antigen overload and chronic antibody responses. Unfortunately, we did not have chance to simultaneously determine avidities of both aCL and antibodies against an infectious antigen. Therefore, we cannot compare avidities of aCL with those against some recall antigen.

Suwannalai et al. [35] reported that the antibody response against autoantigens does not display avidity maturation, as observed both at baseline and at follow-up. A longitudinal examination in our study showed that the avidity of aCLs remained constant during follow-up. Our observation agrees with findings, which have been reported for several of the other autoantibodies. Cucnik et al. [14] who tested a 32 GPI avidity found no significant changes during the 
Table 2. Correlation between levels and avidities of aCL IgG and non-criteria aPLs. Non-criteria aPLs were analyzed in 28 patients

\begin{tabular}{lcccc}
\hline Correlation versus & aß2GPI levels & aPS levels & aPI levels & Anti-phosphatidic acid \\
\hline aCL IgG levels (GPL) & $r=0.77$ & $r=0.91$ & $r=0.89$ & $r=0.92$ \\
& $p<0.0001$ & $p<0.0001$ & $p<0.0001$ & $p<0.0001$ \\
\hline
\end{tabular}

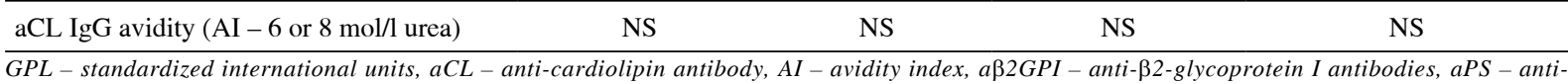
phosphatidylserine antibodies, aPI - anti-phosphatidylinositol antibodies, NS - not significant

course of thrombotic events and pregnancy complications. Similarly, avidities of anti-citrullinated protein antibodies, anti-glutamate decarboxylase antibodies, or those against insulinoma-associated protein 2 did not exhibit extensive avidity maturation [35, 38, 39]. However, fluctuation of avidity has also been described. Avidities of anti-Hu and anti-Yo onconeural antibodies increased or remained invariable during the course of oncological diseases [40].

Only a limited number of previous studies evaluated the avidity of aCLs, eventually aß2GPI, in the relationship to clinical manifestations are available [14, 22-24, 41-43]. Chaotropic agents such as urea or sodium chloride in ELISA have been utilized for avidity determination in these studies. Avidity of aCLs and/or aß2GPI antibodies had been studied in patients with APS and non-APS, including those manifested as thrombosis and obstetric complications [14, 22, 23, 42]. Vlachoyiannopoulos et al. [23] found that the majority of patients with APS produced aPLs with higher avidity than non-APS patients. Predominantly, venous thrombosis was associated with higher avidity of a 32 GPI antibodies [43]. In addition, aCL avidities in patients with autoimmune liver diseases had high avidity, while the avidity of a 32 GPI in patients with HIV infection was lower in comparison with SLE/APS or primary APS patients [22, 24, 25]. However, all these studies evaluated patients with positive aPLs only.

It is known that level and avidity represent important characteristics of antibodies [44]. They are controlled by independent mechanisms. A lack of relationship between aCL IgG levels and avidities in our study does not conflict with this above-mentioned fact. Similarly, Cucnik et al. [43] observed that not all samples with high levels of a 32 GPI corresponded with high avidities.

We demonstrated a strong positive correlation between aCLs and aPLs directed against aß2GPI and other phospholipids. Unfortunately, we had the possibility to analyze this relationship only in patients with positive aCLs. Our findings are in agreement with the conclusions of a recent systematic review stating that the prevalence of all non-criteria aPLs was significantly elevated in APS patients in comparison with those without APS [45]. Nowadays, an examination of certain non-aCL anti-phospholipid antibodies are considered mainly in clinically symptomatic, but for conventional aPLs, seronegative patients [7].

\section{Conclusions}

In summary, we are the first to evaluate aCL IgG avidity in patients not only with high levels, but also with very low ones, which are not included in the APS laboratory criteria. Our results suggested that aCL avidity did not depend on aCL levels and that high-avidity aCLs with potentially deleterious effects might also be present in patients with very low aCL levels. In addition, we confirmed that the avidity of aCLs belongs to the stable characteristics, which changes in time and are not statistically significant. In the future, we plan to extend and define our patients' groups in greater detail and to include a group of healthy participants for further investigation of aCL avidities.

\section{Acknowledgments}

We wish to thank Dr. Karin Malíčková for the selection of serum samples for our avidity analyses and for providing the anti-phospholipid antibody values for patients from the Immunological Department.

This work was supported by the project of Ministry of Health, Czech Republic for conceptual development of research organization RVO-VFN64165/2012 (General University Hospital in Prague, Czech Republic) and by research project of Charles University in Prague (PROGRESS Q25 206025-5).

The authors declare no conflict of interest.

\section{References}

1. Misasi R, Capozzi A, Longo A, et al. (2015): "New" antigenic targets and methodological approaches for refining laboratory diagnosis of antiphospholipid syndrome. J Immunol Res 2015: 858542.

2. Alessandri C, Conti F, Pendolino M, et al. (2011): New autoantigens in the antiphospholipid syndrome. Autoimmun Rev 10: 609-616.

3. Hughes GRV (1983): Thrombosis, abortion, cerebral disease, and the lupus anticoagulant. Br Med J 287: 1088-1089.

4. Hughes GRV (2014): Hughes syndrome/APS. 30 years on, what have we learnt? Opening talk at the 14th International Congress on antiphospholipid antibodies Rio de Janiero, October 2013. Lupus 23: 400-406. 
5. Nikolova-Vlahova MK, Nikolov KV, Baleva MP, et al. (2015): Antiphospholipid antibodies in patients with upper-extremity deep vein thrombosis. Cent Eur J Immunol 40: 307-310.

6. Miyakis S, Lockshin MD, Atsumi T, et al. (2006): International consensus statement on an update of the classification criteria for definite antiphospholipid syndrome (APS). J Thromb Haemost 4: 295-306.

7. Nayfe R, Uthman I, Aoun J, et al. (2013): Seronegative antiphospholipid syndrome. Rheumatology 52: 1358-1367.

8. Forastiero R (2014): Multiple antiphospholipid antibodies positivity and antiphospholipid syndrome criteria re-evaluation. Lupus 23: 1252-1254.

9. Sciascia S, Radin M, Bazzan M, et al. (2017): Novel diagnostic and therapeutic frontiers in thrombotic anti-phospholipid syndrome. Intern Emerg Med 12: 1-7.

10. Bertolaccini ML, Amengual O, Atsumi T, et al. (2011): 'Non-criteria' aPL tests: report of a task force and preconference workshop at the 13th International Congress on Antiphospholipid Antibodies, Galveston, TX, USA, April 2010. Lupus 20: 191-205.

11. Božič B, Kveder T, Rozman B (2007): Affinity and avidity of autoantibodies. In: Shoenfeld Y, Gershwin ME, Meroni PL (eds.). Autoantibodies. Elsevier, Amsterdam: 21-28.

12. Prince HE, Wilson M (2001): Simplified assay for measuring Toxoplasma gondii immunoglobulin $\mathrm{G}$ avidity. Clin Diagn Lab Immunol 8: 904-908.

13. Hazell SL (2007): Clinical utility of avidity assays. Expert Opin Med Diagn 1: 511-519.

14. Cucnik S, Bozic B, Kveder T, et al. (2005): Avidity of anti-beta2-glycoprotein I and thrombosis or pregnancy loss in patients with antiphospholipid syndrome. Ann N Y Acad Sci 1051: 141-147.

15. Cui Z, Zhao MH (2005): Avidity of anti-glomerular basement membrane autoantibodies was associated with disease severity. Clin Immunol 116: 77-82.

16. Suwannalai P, van de Stadt LA, Radner H, et al. (2012): Avidity maturation of anti-citrullinated protein antibodies in rheumatoid arthritis. Arthritis Rheum 64: 1323-1328.

17. Fialová L, Malbohan I, Malickova K (2014): Avidity of anticardiolipin antibodies-A factor that could be important for their detection by ELISA methods. J Appl Biomed 12: 277284.

18. Fialová L, Petrackova M, Kuchar O (2017): Comparison of different enzyme-linked immunosorbent assay methods for avidity determination of antiphospholipid antibodies. J Clin Lab Anal 31: e22121.

19. Fialová L, Bartos A, Svarcova J, et al. (2011): Increased intrathecal high-avidity anti-tau antibodies in patients with multiple sclerosis. PLoS One 6: e27476.

20. Fialová L (2015): Avidity of autoantibodies against antigens in nervous tissue In: Costa A, Villalba E (eds.). Horizons in Neuroscience Research. Nova Science Publishers, New York: 159-169.

21. Fialová L (2016): Affinity and avidity of autoantibodies their clinical significance. Epidemiol Mikrobiol Imunol 65: 155-163.

22. Petrovas C, Vlachoyiannopoulos PG, Kordossis T, et al. (1999): Anti-phospholipid antibodies in HIV infection and SLE with or without anti-phospholipid syndrome: comparisons of phospholipid specificity, avidity and reactivity with beta2-GPI. J Autoimmun 13: 347-355.

23. Vlachoyiannopoulos PG, Petrovas C, Tektonidou M, et al. (1998): Antibodies to beta 2-glycoprotein-I: urea resistance, binding specificity, and association with thrombosis. J Clin Immunol 18: 380-391.

24. Liaskos C, Rigopoulou E, Zachou K, et al. (2005): Prevalence and clinical significance of anticardiolipin antibodies in patients with type 1 autoimmune hepatitis. J Autoimmun 24: 251-260.

25. Zachou K, Liaskos C, Rigopoulou E, et al. (2006): Presence of high avidity anticardiolipin antibodies in patients with autoimmune cholestatic liver diseases. Clin Immunol 119: 203-212.

26. Bertolaccini ML, Amengual O, Andreoli L, et al. (2014): 14th International Congress on Antiphospholipid Antibodies Task Force. Report on antiphospholipid syndrome laboratory diagnostics and trends. Autoimmun Rev 13: 917-930.

27. Carmel-Neiderman NN, Tanne D, Goren I, et al. (2017): Classical and additional antiphospholipid antibodies in blood samples of ischemic stroke patients and healthy controls. Immunol Res 65: 470-476.

28. Almanzar G, Ottensmeier B, Liese J, et al. (2013): Assessment of IgG avidity against pertussis toxin and filamentous hemagglutinin via an adapted enzyme-linked immunosorbent assay (ELISA) using ammonium thiocyanate. J Immunol Methods 387: 36-42.

29. Lackner KJ, MullerCalleja N (2018): Diagnosis and Management of the Antiphospholipid Syndrome. N Engl J Med 379: 1290.

30. Fialová L (2014): Avidity of antiphospholipid antibodies - our current knowledge. Epidemiol Mikrobiol Imunol 63: 221-225.

31. Eisen HN (2014): Affinity enhancement of antibodies: how low-affinity antibodies produced early in immune responses are followed by high-affinity antibodies later and in memory B-cell responses. Cancer Immunol Res 2: 381-392.

32. Dellavance A, Coelho Andrade LE (2014): Immunologic derangement preceding clinical autoimmunity. Lupus 23: 13051308.

33. Krause S, Landherr U, Agardh CD, et al. (2014): GAD autoantibody affinity in adult patients with latent autoimmune diabetes, the study participants of a GAD65 vaccination trial. Diabetes Care 37: 1675-1680.

34. Jianping L, Zhibing Y, Wei Q, et al. (2006): Low avidity and level of serum anti-Abeta antibodies in Alzheimer disease. Alzheimer Dis Assoc Disord 20: 127-132.

35. Suwannalai P, Scherer HU, van der Woude D, et al. (2011): Anti-citrullinated protein antibodies have a low avidity compared with antibodies against recall antigens. Ann Rheum Dis 70: 373-379.

36. Gelderman KA, Drop AC, Trouw LA, et al. (2014): Serum autoantibodies directed against transglutaminase- 2 have a low avidity compared with alloantibodies against gliadin in coeliac disease. Clin Exp Immunol 177: 86-93.

37. van Delft MAM, Verheul MK, Burgers LE, et al. (2018): The anti-carbamylated protein antibody response is of overall low avidity despite extensive isotype switching. Rheumatology 57: 1583-1591.

38. Mayr A, Schlosser M, Grober N, et al. (2007): GAD autoantibody affinity and epitope specificity identify distinct immunization profiles in children at risk for type 1 diabetes. Diabetes 56: 1527-1533.

39. Krause S, Chmiel R, Bonifacio E, et al. (2012): IA-2 autoantibody affinity in children at risk for type 1 diabetes. Clin Immunol 145: 224-229. 
40. Totland C, Aarseth J, Vedeler C (2007): Hu and Yo antibodies have heterogeneous avidity. J Neuroimmunol 185: 162-167.

41. Bozic B, Cucnik S, Kveder T, et al. (2005): Avidity of anti-beta-2-glycoprotein I antibodies. Autoimmun Rev 4: 303308.

42. Cucnik S, Kveder T, Artenjak A, et al. (2012): Avidity of anti-beta2-glycoprotein I antibodies in patients with antiphospholipid syndrome. Lupus 21: 764-765.

43. Cucnik S, Kveder T, Ulcova-Gallova Z, et al. (2011): The avidity of anti-beta2-glycoprotein I antibodies in patients with or without antiphospholipid syndrome: a collaborative study in the frame of the European forum on antiphospholipid antibodies. Lupus 20: 1166-1171.

44. Steward MW (1979): Antibody affinity: immunogenetic aspects and relationship to immune complex disease. J Clin Pathol Suppl 13: 120-125.

45. Rodriguez-Garcia V, Ioannou Y, Fernandez-Nebro A, et al. (2015): Examining the prevalence of non-criteria anti-phospholipid antibodies in patients with anti-phospholipid syndrome: a systematic review. Rheumatology 54: 2042-2050. 